\title{
Determinant Bounds and the Matsubara UV Problem of Many-Fermion Systems
}

\author{
Walter A. de S. Pedra ${ }^{1,2,3}$, Manfred Salmhofer ${ }^{1,2}$ \\ 1 Theoretical Physics, University of Leipzig, Postfach 100920, 04009 Leipzig, Germany. \\ E-mail: salmhofer@itp.uni-leipzig.de \\ 2 Max-Planck Institute for Mathematics in the Sciences, Inselstr. 22, 04103 Leipzig, Germany \\ 3 Institut für Mathematik, Universität Mainz, D-55128 Mainz, Germany
}

Received: 18 July 2007 / Accepted: 17 December 2007

Published online: 6 May 2008 - (C) Springer-Verlag 2008

\section{Dedicated to Jürg Fröhlich in celebration of his $61^{\text {st }}$ birthday}

\begin{abstract}
It is known that perturbation theory converges in fermionic field theory at weak coupling if the interaction and the covariance are summable and if certain determinants arising in the expansion can be bounded efficiently, e.g. if the covariance admits a Gram representation with a finite Gram constant. The covariances of the standard manyfermion systems do not fall into this class due to the slow decay of the covariance at large Matsubara frequency, giving rise to a UV problem in the integration over degrees of freedom with Matsubara frequencies larger than some $\Omega$ (usually the first step in a multiscale analysis). We show that these covariances do not have Gram representations on any separable Hilbert space. We then prove a general bound for determinants associated to chronological products which is stronger than the usual Gram bound and which applies to the many-fermion case. This allows us to prove convergence of the first integration step in a rather easy way, for a short-range interaction which can be arbitrarily strong, provided $\Omega$ is chosen large enough. Moreover, we give - for the first time - nonperturbative bounds on all scales for the case of scale decompositions of the propagator which do not impose cutoffs on the Matsubara frequency.
\end{abstract}

\section{Gram Representations and Determinant Bounds}

Let $\mathbb{X}$ be a set and $M: \mathbb{X}^{2} \rightarrow \mathbb{C},(x, y) \mapsto M(x, y)$. We call $M$ an $(\mathbb{X} \times \mathbb{X})$-matrix and use the notation $M=\left(M_{x y}\right)_{x, y \in \mathbb{X}}$ (if $\mathbb{X}=\{1, \ldots, n\}$, we call it as usual an $(n \times n)$-matrix).

Definition 1.1. Let $M$ be an $(\mathbb{X} \times \mathbb{X})$-matrix. A triple $(\mathcal{H}, v, w)$, where $\mathcal{H}$ is a Hilbert space and $v$ and $w$ are maps from $\mathbb{X}$ to $\mathcal{H}$, is called a Gram representation of $M$ if

$$
\forall x, x^{\prime} \in \mathbb{X}: \quad M_{x x^{\prime}}=\left\langle v_{x}, w_{x^{\prime}}\right\rangle,
$$

and if there is a finite constant $\gamma_{M}>0$ such that

$$
\sup _{x, x^{\prime} \in \mathbb{X}}\left\|v_{x}\right\|\left\|w_{x^{\prime}}\right\| \leq \gamma_{M}^{2}
$$


$\gamma_{M}$ is called the Gram constant of $M$ associated to the Gram representation $(\mathcal{H}, v, w)$.

If $M$ has a Gram representation, then the Gram estimate (see, e.g., Lemma B.30 of [S98b]) implies that for all $n \in \mathbb{N}$ and all $x_{1}, \ldots, x_{n}, y_{1}, \ldots, y_{n} \in \mathbb{X}$,

$$
\left|\operatorname{det}\left[\left(M_{x_{k} y_{l}}\right)_{k, l}\right]\right| \leq \prod_{k=1}^{n}\left\|v_{x_{k}}\right\|\left\|w_{y_{k}}\right\| \leq \gamma_{M}^{2 n} .
$$

Every $(n \times n)$-matrix $A$ has a Gram representation - the equation $A=1 \cdot A$ (where 1 denotes the unit matrix) means that $A_{k l}=\left\langle e_{k}, a_{l}\right\rangle_{\mathbb{C}^{n}}$, where $e_{k}$ is the $k^{\text {th }}$ row of 1 and $a_{l}$ is the $l^{\text {th }}$ column of $A$. The associated Gram estimate $|\operatorname{det} A| \leq \prod_{l}\left\|a_{l}\right\|_{2}$, the Hadamard bound, has associated Gram constant $\gamma_{\mathrm{Had}}^{2}=\max _{l}\left\|a_{l}\right\|_{2}$. Although considering diagonal matrices shows that the Hadamard bound is optimal, the way it was derived here is basis-dependent, and its application in an arbitrary basis can lead to a significant overestimate of the determinant. For instance, the matrix $P=v \otimes v$, where $v=(1, \ldots, 1)^{T} \in \mathbb{C}^{n}$ has $P_{k l}=1$ for all $k, l$, so the above Gram representation gives $\gamma_{\text {Had }}^{2}=\sqrt{n}$, thus the bound $|\operatorname{det} P| \leq n^{n / 2}$. On the other hand, $P$ has the Gram representation $P_{i j}=w \cdot w^{T}$ with $w=n^{-1 / 2}(1, \ldots, 1)$, which gives the bound $\operatorname{det} P \leq 1$.

Thus the main issue about Gram bounds for a given class of matrices is not the existence of some bound, but its size, and its dependence on $n$. Specifically, what is really needed in the proof of convergence of fermionic perturbation theory given in [SW] are bounds of the following type: there is a finite constant $\delta$ such that for all $n \in \mathbb{N}$ and all $x_{1}, \ldots, x_{n}, y_{1}, \ldots, y_{n} \in \mathbb{X}$,

$$
\sup _{P \in \mathcal{P}_{n, 1}}\left|\operatorname{det}\left(C_{x_{i} y_{j}} P_{i j}\right)_{i, j}\right| \leq \delta^{2 n} .
$$

Here $\mathcal{P}_{n, 1}$ denotes the set of complex hermitian $(n \times n)$-matrices $P=\left(P_{i j}\right)$ that are nonnegative, i.e. $\sum_{i, j=1}^{n} P_{i j} \bar{c}_{i} c_{j} \geq 0$ for all $c_{1}, \ldots, c_{n} \in \mathbb{C}$, and that have diagonal elements $P_{i i} \leq 1$. Such matrices $P$ arise naturally in interpolation constructions of the tree expansion for the connected functions; they are positive if the tree expansion is chosen well [AR, SW].

We briefly recall Lemmas 7 and 8 of [SW]: The positivity of $P$ implies that $P=Q^{2}=Q^{*} Q$ with $Q \geq 0$, i.e.

$$
P_{i j}=\left\langle q_{i}, q_{j}\right\rangle,
$$

where $q_{i}$ is the $i^{\text {th }}$ column of $Q$. Because $\left\langle q_{i}, q_{i}\right\rangle=P_{i i} \leq 1$ the Gram constant of $P$ is bounded by 1 . If $C$ has a Gram representation $(\mathcal{H}, v, w)$, then the matrix with elements $M_{i j}=C_{x_{i} y_{j}} P_{i j}$ has a Gram representation

$$
M_{i j}=\left\langle v_{x_{i}} \otimes q_{i}, w_{y_{j}} \otimes q_{j}\right\rangle,
$$

and $M$ has the same Gram constant as $C$ because $\left\|q_{i}\right\| \leq 1$ for all $i$.

Definition 1.2. Let $C$ be an $(\mathbb{X} \times \mathbb{X})$-matrix. A finite constant $\delta_{C}>0$ is called a determinant bound of $C$ if for all $n \in \mathbb{N}$ and all $x_{1}, \ldots, x_{n}, y_{1}, \ldots, y_{n} \in \mathbb{X}$,

$$
\sup _{p_{1}, \ldots, p_{n}, q_{1}, \ldots, q_{n} \in B_{1}^{(n)}}\left|\operatorname{det}\left(\left\langle p_{i}, q_{j}\right\rangle C_{x_{i} y_{j}}\right)_{i, j}\right| \leq \delta_{C}^{2 n} .
$$

Here $B_{1}^{(n)}=\left\{\xi \in \mathbb{C}^{n}:\|\xi\|_{2} \leq 1\right\}$ denotes the closed $n$-dimensional unit ball. 
We have replaced the supremum over $P \in \mathcal{P}_{n, 1}$ by that over a larger set in Definition 1.2 because this makes the definition robust under the operation of taking arbitrary submatrices (positivity is spoiled by that operation).

If $C$ has a Gram representation with Gram constant $\gamma_{C}$, then $C$ also has a determinant bound $\delta_{C}=\gamma_{C}$ by the same argument as above, i.e. writing $\left\langle p_{i}, q_{j}\right\rangle C_{x_{i} y_{j}}=$ $\left\langle p_{i} \otimes v_{x_{i}}, q_{j} \otimes w_{y_{j}}\right\rangle$. However, the Gram representation is not necessary for a useful determinant bound, and in this paper, we prove optimal determinant bounds for a class of covariance matrices for which no Gram representation with a good Gram constant is known. As will be discussed in Sect. 2, these matrices arise naturally in time-ordered perturbation theory and standard functional integral representations of many-fermion systems. The constructions we give are motivated by similar ones in [FKT04], and we shall discuss this relation in more detail in Sect. 2.

Theorem 1.3. Let $K, k \in \mathbb{N}_{0}, k+K \geq 1$, and $C_{0}, \ldots, C_{k+K}$ be $(\mathbb{X} \times \mathbb{X})$-matrices. Assume that for all $l \in\{0, \ldots, k+K\}, C_{l}$ has a Gram representation with Gram constant $\gamma_{l}$. Let $(\mathcal{J}, \succ)$ be a totally ordered set, and for all $l \in\{1, \ldots, k+K\}$ let $\varphi_{l}$ and $\varphi_{l}^{\prime}$ be functions from $\mathbb{X}$ to $\mathcal{J}$. Denote $1_{A}=1$ if $A$ is true and $1_{A}=0$ otherwise. Then the $(\mathbb{X} \times \mathbb{X})$-matrix $M$ given by

$$
M_{x y}=\left(C_{0}\right)_{x y}+\sum_{l=1}^{k}\left(C_{l}\right)_{x y} 1_{\varphi_{l}^{\prime}(x) \succ \varphi_{l}(y)}+\sum_{l=k+1}^{k+K}\left(C_{l}\right)_{x y} 1_{\varphi_{l}^{\prime}(x) \succeq \varphi_{l}(y)}
$$

has determinant bound $\delta_{M}=\sum_{l=0}^{k+K} \gamma_{l}$.

Theorem 1.3 is proven in Sect. 3 .

The bound given in Theorem 1.3 is optimal in the following sense. Let us assume that for each $l$, the Gram representation for the $C_{l}$ is optimal in that the Gram constant $\gamma_{C_{l}}$ satisfies $\gamma_{C_{l}}^{2}=\sup _{x, y \in \mathbb{X}}\left|\left(C_{l}\right)_{x y}\right|$, and that the decomposition (8) is nonredundant in the sense that for any choice of $x$ and $y$, only one of the summands is nonzero (in particular, $C_{0}=0$ ). Then the determinant bound given in Theorem 1.3 is optimal up to a factor $k+K$ because

$$
\delta_{M} \leq(k+K)\left(\sup _{x, y \in \mathbb{X}}\left|M_{x y}\right|\right)^{\frac{1}{2}}
$$

and because, by Definition 1.2, the determinant bound $\delta_{M}$ of a matrix $M$ satisfies

$$
\delta_{M}^{2 n} \geq \sup _{\substack{x_{1}, \ldots, x_{n} \in \mathbb{X} \\ y_{1}, \ldots, y_{n} \in \mathbb{X}}}\left|\operatorname{det}\left(M_{x_{i} y_{j}} \delta_{i j}\right)\right|=\left(\sup _{x, y \in \mathbb{X}}\left|M_{x y}\right|\right)^{n} .
$$

\section{The Matsubara UV Problem for Fermion Systems}

In this section, we specify the covariances for the many-fermion models, and then briefly review the well-known problem with the standard Gram representation due to the slow decay at large frequencies which is caused by the indicator functions from time ordering, which are special cases of the ones appearing in (8) (the Matsubara UV problem). We show that, if a Gram representation of these covariances exists, it has rather unusual properties. Then we state our main results for these models which follow directly 
from our new determinant bounds. A detailed analysis of these models will be given in $[\mathrm{PS}]$.

We consider the standard many-fermion model, as formulated for instance in [BR] or in [S98b], Chapter 4. The Hamiltonian of this model is of the form $H=H_{0}+V$. The free part $H_{0}$ is given by a hopping term (if a lattice model is considered) or a differential operator (if a continuum model is considered). In either case, the relevant data for the present discussion are a momentum space $\mathcal{B}$ dual to configuration space $X$ and an energy function $E: \mathcal{B} \rightarrow \mathbb{R}, \mathrm{p} \mapsto E(\mathrm{p})$, which assigns an energy $E(\mathrm{p})$ to a particle with (quasi)momentum $\mathrm{p} \in \mathcal{B}$. The interaction part $V$ of $H$ describes the interaction of two or more particles (see below).

To be specific, we review briefly how $E$ arises in some relevant cases. For a continuum system in $d$ spatial dimensions without a crystal potential, $X=\mathbb{R}^{d}, \mathcal{B}=\mathbb{R}^{d}$, and $E(\mathrm{p})=\mathrm{p}^{2}-\mu$, where the parameter $\mu>0$, the chemical potential, is a Lagrange parameter used to adjust the particle density. Particles in a crystal are modelled by a periodic Schrödinger operator containing a potential that is $\Gamma$-periodic, where $\Gamma \subset \mathbb{R}^{d}$ is a lattice of maximal rank. In this case, $\mathcal{B}$ is the torus $\mathcal{B}=\mathbb{R}^{d} / \Gamma^{\#}$, where $\Gamma^{\#}$ is the dual lattice to $\Gamma$. The operator has a band spectrum $p \mapsto\left(e_{v}(\mathrm{p})\right)_{v \in \mathbb{N}}$, with the index $v$ labelling the bands. The case of a single $E$ is obtained by restricting to a single band $v=v_{0}$ and setting $E(\mathrm{p})=e_{v_{0}}(\mathrm{p})-\mu$. For a (one-band) lattice model on a spatial lattice $\Lambda, \mathcal{B}=\mathbb{R}^{d} / \Lambda^{\#}$ is again a torus, and $E(\mathrm{p})$ is the Fourier transform of the hopping matrix (see [S98b], Chapter 4). The motivation for restricting to a single band is that the interesting case is the one where $E(\mathrm{p})$ has a nontrivial zero set, and that in many interesting cases, the bands do not overlap, so that for this zero set, only a single band matters.

In field theoretic constructions, one often considers configuration spaces $X=\Gamma / L \Gamma$ that have sidelengths $L \in \mathbb{N}$, hence finite volume, in which case momentum space is discrete: $\mathcal{B}=\mathcal{B}_{L}=L^{-1} \Gamma^{\#} / \Gamma^{\#}$. We shall consider the cases of finite and infinite volume in parallel and use the conventions of [S98b], Appendix A, for the Fourier transform. We denote by $\mu(\mathrm{dp})$ the natural invariant Haar measure on the torus $\mathcal{B}$; specifically, for the continuous torus corresponding to infinite volume it is given by $(2 \pi)^{-d}$ times Lebesgue measure, for the discrete torus $\mathcal{B}_{L}$ corresponding to a finite volume it is given by the inverse of the volume times the counting measure. We shall drop the subscript $L$ on $\mathcal{B}_{L}$ when no confusion can arise.

The interaction part of the Hamiltonian is assumed to be given by a two-body potential $v$, where $v(x-y)$ is the interaction energy of a configuration with one particle at $x$ and one particle at $y$. Most of the present paper is concerned with properties of the covariance, in which the interaction plays no role. However, the decay properties of the interaction are important for convergence of expansions, see below. The correct treatment of the interaction is difficult, but some progress has been made by multiscale expansion methods. One of the purposes of the present paper is to simplify and extend parts of this analysis, namely the ultraviolet (UV) integration, which is quite different from the analysis of the infrared singularity which arises in the limit of zero temperature.

We briefly discuss the UV problems arising in such models. There is a spatial UV problem associated to continuum interactions that have a singularity at coinciding points, such as, for instance, a Yukawa potential $\mathrm{e}^{-\alpha|x|} /|x|$, but this is not the issue we address here.

There are also different UV problems associated to the covariances. The first one is related to the noncompactness of momentum space in the first example mentioned above. A similar problem arises for the periodic Schrödinger operator, namely there is 
an infinite number of bands. For the lattice system, the lattice spacing provides a natural spatial ultraviolet cutoff. The UV problem we are concerned with here is the discontinuity of the covariance as a function of the time variable, and the corresponding slow decay of its Fourier transform in the dual variable, the Matsubara frequency. In the continuum case $X=\mathbb{R}^{d}$, we shall therefore impose a cutoff on the spatial part of momentum. We do this by using the measure $\mu^{a}$ where, for $a>0, \mu^{a}(\mathrm{dp})=\chi(a \mathrm{p}) \mu(\mathrm{dp})$, with $\chi$ a nonnegative function on $\mathbb{R}^{d}$ of compact support chosen such that $\int \chi(\mathrm{p}) \mu(\mathrm{dp})=1$, hence $\mu^{a}(\mathcal{B})=a^{-d}$. The UV cutoff parameter $a$ scales similarly to a lattice spacing: if $X=a \mathbb{Z}^{d}, \mu(\mathcal{B})=a^{-d}$. For a general lattice $\Gamma$, which may have different spacings in the different directions, we define $a$ by $\mu(\mathcal{B})=a^{-d}$, so that $a$ is a geometric mean of the lattice spacings, and set $\mu^{a}=\mu$.

Let $\beta>0, f_{\beta}(E)=\left(1+\mathrm{e}^{\beta E}\right)^{-1}$, and for $(\tau, E) \in(-\beta, \beta] \times \mathbb{R}$ let

$$
\mathbb{C}(\tau, E)= \begin{cases}-\mathrm{e}^{-\tau E}\left(1-f_{\beta}(E)\right) & \text { for } 0<\tau \leq \beta \\ \mathrm{e}^{-\tau E} f_{\beta}(E) & \text { for }-\beta<\tau \leq 0 .\end{cases}
$$

Extend the function $\mathbb{C}$ to a function on $\mathbb{R} \times \mathbb{R}$ that is $2 \beta$ - periodic in $\tau$. Note that

$$
\mathbb{C}(\tau+\beta, E)=-\mathbb{C}(\tau, E) .
$$

In the application, the parameter $\beta$ is the inverse temperature, and the Fermi function $f_{\beta}$ is the expected occupation number for free fermions.

Definition 2.1. The free covariance (free one-particle Green function) for a manyfermion system is the inverse Fourier transform of the map $\mathrm{p} \mapsto C(\tau, E(\mathrm{p}))$ :

$$
C_{(\tau, \mathrm{x}),\left(\tau^{\prime}, \mathrm{x}^{\prime}\right)}=\int_{\mathcal{B}} \mu^{a}(\mathrm{dp}) \mathrm{e}^{\mathrm{ip} \cdot\left(\mathrm{x}-\mathrm{x}^{\prime}\right)} \mathbb{C}\left(\tau-\tau^{\prime}, E(\mathrm{p})\right) .
$$

More generally, let $\mathbf{h} \in L^{1}\left(\mathcal{B}, \mu^{a}\right)$ and define

$$
C_{(\tau, \mathrm{x}),\left(\tau^{\prime}, \mathrm{x}^{\prime}\right)}^{(\mathbf{h})}=\int_{\mathcal{B}} \mu^{a}(\mathrm{dp}) \mathbf{h}(p) \mathrm{e}^{\mathrm{ip} \cdot\left(\mathrm{x}-\mathrm{x}^{\prime}\right)} \mathbb{C}\left(\tau-\tau^{\prime}, E(\mathrm{p})\right) .
$$

The function (13) arises in time-ordered expansions relative to a quasifree state corresponding to a quadratic Hamiltonian $H_{0}$ with dispersion relation $E$, as discussed above. If we denote the fermionic field operators in a second-quantized formulation by $a_{\mathrm{x}}$ and set $a_{\tau, \mathrm{x}}^{(+)}=\mathrm{e}^{\tau H_{0}} a_{\mathrm{x}}^{*} \mathrm{e}^{-\tau H_{0}}$ and $a_{\tau, \mathrm{x}}^{(-)}=\mathrm{e}^{\tau H_{0}} a_{\mathrm{x}} \mathrm{e}^{-\tau H_{0}}$,

$$
C_{(\tau, \mathrm{x}),\left(\tau^{\prime}, \mathrm{x}^{\prime}\right)}=-\omega_{0}\left(\mathbb{T}\left[a_{\tau, \mathrm{x}}^{(-)} a_{\tau^{\prime}, \mathrm{x}^{\prime}}^{(+)}\right]\right)
$$

where $\omega_{0}$ denotes the quasifree state corresponding to $H_{0}$ and to the inverse temperature $\beta$, around which we expand, and $\mathbb{T}$ denotes time ordering [AGD]. As $\omega_{0}$ is a KMS state, (15) makes sense for all $\tau, \tau^{\prime} \in \mathbb{R}$ with $0 \leq\left|\tau-\tau^{\prime}\right| \leq \beta$. Because the field operators obey the canonical anticommutation relations, the time ordering, which avoids commutator terms (keeping only the fermionic antisymmetry), leads to discontinuities in the function, which are explicit in (11). Thus the discontinuity of $C$ reflects the microscopic structure of the physical system, as encoded in the anticommutation relations of the field operators that generate the observable algebra.

In the above definitions, we have assumed for simplicity that $C_{x y}$ and $v(x-y)$ depend only on space coordinates $x, y \in X$, with $X$ as above. It is straightforward to generalize 
our arguments to the case with spin or additional indices on which the fields depend (e.g. for the usual models with $S U(N)$ symmetry, this just amounts to replacing $C$ by $C \otimes 1_{N}$, where $1_{N}$ denotes the $N$-dimensional unit matrix, and the representations by inner products used below can be adapted in the obvious way by tensoring with a factor $\mathbb{C}^{N}$ and using that $\delta_{i, j}=\left\langle e_{i}, e_{j}\right\rangle$ for any orthonormal basis of $\left.\mathbb{C}^{N}\right)$.

Obviously, (13) can be regarded as defining an $\left(\mathbb{X}_{d} \times \mathbb{X}_{d}\right)$-matrix, where

$$
\mathbb{X}_{d}=[0, \beta) \times X \text {. }
$$

Let

$$
\hat{\mathbb{X}}_{d}=\mathbb{M}_{F} \times \mathcal{B}
$$

where $\mathbb{M}_{F}=\frac{\pi}{\beta}(2 \mathbb{Z}+1)$. The Fourier transform of $C$ is

$$
\hat{C}(\omega, \mathrm{p})=\frac{1}{\mathrm{i} \omega-E(\mathrm{p})}, \quad(\omega, \mathrm{p}) \in \hat{\mathbb{X}}_{d} .
$$

The standard way to obtain a Gram representation for (regularized) covariances in quantum field theory is via their Fourier representation. In our present setting, if $\hat{D} \in L^{1}\left(\hat{\mathbb{X}}_{d}\right)$, then a Gram representation for $D$ is obtained simply by setting $\mathcal{H}=L^{2}\left(\hat{\mathbb{X}}_{d}\right)$, and for $(\tau, \mathrm{x}) \in \mathbb{X}_{d}$,

$$
\begin{aligned}
v_{\tau, \mathrm{x}}(\omega, \mathrm{p}) & =\mathrm{e}^{-\mathrm{i} \tau \omega+\mathrm{ip} \cdot \mathrm{x}}|\hat{D}(\omega, \mathrm{p})|^{1 / 2}, \\
w_{\tau, \mathrm{x}}(\omega, \mathrm{p}) & =\mathrm{e}^{-\mathrm{i} \tau \omega+\mathrm{ip} \cdot \mathrm{x}}|\hat{D}(\omega, \mathrm{p})|^{-1 / 2} \hat{D}(\omega, \mathrm{p}) .
\end{aligned}
$$

The Gram constant is $\gamma_{D}=\|\hat{D}\|_{1}$, and the dominated convergence theorem implies continuity of the maps $(\tau, \mathrm{x}) \mapsto v_{\tau, \mathrm{x}}$ and $(\tau, \mathrm{x}) \mapsto w_{\tau, \mathrm{x}}$.

However, the $\hat{C}$ in (18) decays so slowly as a function of the Matsubara frequency $\omega$ that $\hat{C} \notin L^{1}\left(\hat{\mathbb{X}}_{d}\right)$ (this must be so because $\mathbb{C}$ itself has a discontinuity, so its Fourier transform cannot be integrable). Thus the standard procedure to obtain a Gram representation fails.

Lemma 2.2. Let $U$ be the $(\mathbb{R} \times \mathbb{R})$-matrix given by

$$
U_{s t}=\left\{\begin{array}{ll}
1, & s \geq t \\
0, & s<t
\end{array} .\right.
$$

If $(\mathcal{H}, v, w)$ is a Gram representation of $U$, then $\mathcal{H}$ is non-separable and the maps $t \mapsto v_{t}$ and $t \mapsto w_{t}$ are discontinuous at all $t \in \mathbb{R}$.

Proof. For all $s, t \in \mathbb{R}, U_{s t}=\left\langle v_{s}, w_{t}\right\rangle$, so for $t^{\prime}>t,\left\langle v_{t}, w_{t}-w_{t^{\prime}}\right\rangle=1$ and for $t^{\prime}<t$, $\left\langle v_{t^{\prime}}, w_{t}-w_{t^{\prime}}\right\rangle=-1$. Thus, by the Schwarz inequality and the bound $\sup _{t}\left\|v_{t}\right\| \leq \gamma_{U}$,

$$
\forall t, t^{\prime}: t \neq t^{\prime} \Longrightarrow\left\|w_{t}-w_{t^{\prime}}\right\| \geq \frac{1}{\gamma_{U}} .
$$

Thus the map $t \rightarrow w_{t}$ is discontinuous everywhere. Reversing the roles of $v_{t}$ and $w_{t}$ in the above argument implies the same for the map $t \rightarrow v_{t}$. An obvious variant of this argument implies discontinuity in the weak topology as well. Set $W=\left\{w_{t}: t \in \mathbb{R}\right\}$. Let $A \subset \mathcal{H}$ be countable. For all $x \in A$, Eq. (21) and the triangle inequality imply that $\left\{y \in \mathcal{H}:\|y-x\|<\frac{1}{4 \gamma_{U}}\right\}$ contains at most one element of $W$. Thus the $\frac{1}{4 \gamma_{U}}-$ neighbourhood of $A$ contains only countably many elements of $W$, hence $A$ is not dense in $\mathcal{H}$. 
Corollary 2.3. The covariance matrix of the many-fermion system given by (13) has no Gram representation on a separable Hilbert space.

Proof. The function $\tau \mapsto \mathbb{D}(\tau, E)=\mathbb{C}(\tau, E)-\mathbb{C}(\tau, 0)$ is continuous in $\tau$. Its Fourier transform,

$$
\omega \mapsto-\frac{E}{\mathrm{i} \omega(\mathrm{i} \omega-E)},
$$

is in $\ell^{1}$. Thus

$$
D_{(\tau, \mathrm{x}),\left(\tau^{\prime}, \mathrm{x}^{\prime}\right)}=\int \mu^{a}(\mathrm{~d} p) \mathrm{e}^{\mathrm{ip} \cdot\left(\mathrm{x}-\mathrm{x}^{\prime}\right)} \mathbb{D}\left(\tau-\tau^{\prime}, E(\mathrm{p})\right)
$$

has the Gram representation given in (19). An elementary argument involving direct sums of Hilbert spaces shows that $C=D+D^{\prime}$ has a Gram representation if and only if $D^{\prime}$ has a Gram representation. Assume that $C$, given by (13), has a Gram representation on a separable Hilbert space $\mathcal{H}$. Then $C-D$ has a Gram representation on a direct sum of separable Hilbert spaces, which is itself separable. But $C-D$ is

$$
\delta_{\mathrm{x}, \mathrm{x}^{\prime}}^{a}\left(U_{\tau, \tau^{\prime}}-\frac{1}{2}\right)
$$

with $\delta_{\mathrm{x}, \mathrm{x}^{\prime}}^{a}=\int \mu^{a}(\mathrm{~d} p) \mathrm{e}^{\mathrm{ip} \cdot\left(\mathrm{x}-\mathrm{x}^{\prime}\right)}$ and $U$ as in Lemma 2.2, which has no Gram representation on any separable Hilbert space.

Our main use of Gram representations is, of course, to bound determinants of the type occurring in (4). Lemma 2.2 does not exclude that a useful Gram representation, i.e. one with a good Gram constant, can be found, but it shows that the representation will be very different from the ones used so far in fermion models, which all involve separable Hilbert spaces and where continuity of the maps $v$ and $w$ holds.

One can attempt to circumvent the above problem by introducing a UV cutoff $\Omega>0$, which restricts the sum over frequencies $\omega$ to a finite set (for instance by regularizing to $\hat{\mathbb{C}}_{\chi}(\omega, \mathrm{p})=\hat{\mathbb{C}}(\omega, \mathrm{p}) \chi(\omega / \Omega)$, where $\chi$ is a smooth function of compact support). This obviously makes the standard Gram constant finite, Of course, a UV cutoff cannot simply be imposed, because it implies that the time-ordered imaginary-time correlation functions are continuous and therefore not physical. The Gram constant $\gamma_{\mathbb{C}_{\chi}} \sim \log \Omega$ diverges for $\Omega \rightarrow \infty$. One can attempt to perform the limit $\Omega \rightarrow \infty$ by multiscale and renormalization techniques. The approach via determinant bounds developed in the next sections is, however, much simpler and more natural that such a multiscale approach, and it makes the latter unnecessary.

Recall that momentum space is $\mathcal{B}=\mathbb{R}^{d}$ for a continuous system and $\mathcal{B}=\mathbb{R}^{d} / \Gamma^{\#}$ for a system on a lattice $\Gamma$, that in the continuum case, $\mu^{a}$ contains an ultraviolet cutoff, and that $\mathcal{B}_{L}=L^{-1} \Gamma^{\#} / \Gamma^{\#}$ is the corresponding momentum space for the finite-volume system. The main result about the determinant bound of many-fermion covariances is as follows.

Theorem 2.4. Let $E: \mathcal{B} \rightarrow \mathbb{R}$ be bounded and measurable. Then the fermionic covariance matrix $C^{(\mathbf{h})}$ given in (14) has determinant bound

$$
\delta_{C^{(\mathbf{h})}}=2\left(\int \mu^{a}(\mathrm{dp})|\mathbf{h}(\mathrm{p})|\right)^{1 / 2} .
$$

In particular, the covariance $C$ defined in (13) has $\delta_{C}=2 \mu^{a}(\mathcal{B})^{1 / 2}$. 
Theorem 2.4 is proven after Corollary 4.2. As mentioned after Theorem 1.3, this bound is optimal up to the prefactor 2 .

In Sect. 4, we discuss the decay constant of these covariances and prove a convergence theorem for the expansion for the fermionic effective action.

In Sect. 5, we discuss the properties of covariances obtained by a splitting into small and large frequencies and prove that the integration over fields with large frequencies, which usually is the first step in a multiscale treatment, is given by convergent expansions, for arbitrarily large initial interaction strength.

When rewriting traces using Trotter-type formulas, to obtain functional integral representations, one typically obtains time-discretized covariances. The bounds given here apply to them as well, uniformly in the parameter $n$ that defines the discretization [PS].

\section{Determinants and Chronological Products}

In this section we show that determinants corresponding to a general chronological ordering have good determinant bounds and prove Theorem 1.3. We first recall some standard facts and fix notation.

Definition 3.1. Let $V$ be a finite-dimensional vector space over $\mathbb{C}$.

1. Let $k \in \mathbb{N}$. A totally antisymmetric $k$-linear map $\alpha: V^{k} \rightarrow \mathbb{C}$ is called $\boldsymbol{k}$-form. The vector space of all $k$-forms is identified with $\bigwedge^{k} V^{*}$. We also set $\bigwedge^{0} V^{*}=\mathbb{C}$.

2. Let $k, l \in \mathbb{N}$. The exterior product of $\alpha \in \bigwedge^{k} V^{*}$ and $\beta \in \bigwedge^{l} V^{*}, \alpha \wedge \beta \in \bigwedge^{k+l} V^{*}$, acts on $v_{1}, \ldots, v_{k+l} \in V$ as

$$
\begin{aligned}
(\alpha & \wedge \beta)\left(v_{1}, \ldots, v_{k+l}\right) \\
= & \frac{1}{k ! l !} \sum_{\sigma \in S_{k+l}} \operatorname{sgn}(\sigma) \alpha\left(v_{\sigma(1)}, \ldots, v_{\sigma(k)}\right) \beta\left(v_{\sigma(k+1)}, \ldots, v_{\sigma(k+l)}\right) .
\end{aligned}
$$

Here $S_{n}$ denotes the set of permutations of $\{1, \ldots, n\}$.

The exterior algebra $\bigwedge V^{*}$ over the vector space $V$ is

$$
\bigwedge V^{*}=\bigoplus_{k=0}^{\infty} \bigwedge^{k} V^{*}
$$

We identify $\bigwedge V$ with $\bigwedge V^{* *}$, the exterior algebra over $V^{*}$.

The following condition defines a duality between the spaces $\bigwedge^{k} V^{*}$ and $\bigwedge^{k} V$ : for $\alpha=\alpha_{1} \wedge \ldots \wedge \alpha_{k} \in \wedge^{k} V^{*}$ and $v=v_{1} \wedge \ldots \wedge v_{k} \in \bigwedge^{k} V$,

$$
\langle\alpha, v\rangle=\operatorname{det}\left(\alpha_{i}\left(v_{j}\right)\right)_{i, j}
$$

This duality defines a vector space isomorphism $\bigwedge^{k} V^{*} \rightarrow\left(\bigwedge^{k} V\right)^{*}$ :

$$
\left\langle\alpha_{1} \wedge \ldots \wedge \alpha_{k}, v_{1} \wedge \ldots \wedge v_{k}\right\rangle=\alpha_{1} \wedge \ldots \wedge \alpha_{k}\left(v_{1}, \ldots, v_{k}\right)
$$

(this isomorphism is unique only up to a multiplicative factor, and different conventions are used in the literature). Finally, the isomorphisms (29), $k \in \mathbb{N}$, canonically induce an isomorphism between $\bigwedge V^{*}$ and $(\bigwedge V)^{*}$. 
Definition 3.2. Let End $\bigwedge V^{*}$ denote the set of endomorphisms of $\bigwedge V^{*}$.

1. For $w \in \bigwedge V$ define $w\lrcorner \in$ End $\bigwedge V^{*}$ by the condition

$$
\forall v \in \bigwedge V:\langle w\lrcorner \alpha, v\rangle=\langle\alpha, w \wedge v\rangle
$$

2. For $\alpha \in V^{*}$ let $(\alpha \wedge) \in$ End $\bigwedge V^{*}$ be defined by

$$
\forall \beta \in \bigwedge V^{*}: \quad(\alpha \wedge): \beta \mapsto \alpha \wedge \beta
$$

Lemma 3.3. These endomorphisms obey canonical anticommutation relations:

1. $\left(\alpha_{1} \wedge\right)\left(\alpha_{2} \wedge\right)+\left(\alpha_{2} \wedge\right)\left(\alpha_{1} \wedge\right)=0$, for all $\alpha_{1}, \alpha_{2} \in V^{*}$.

2. $\left.\left.\left.\left.u_{1}\right\lrcorner u_{2}\right\lrcorner+u_{2}\right\lrcorner u_{1}\right\lrcorner=0$, for all $u_{1}, u_{2} \in V$.

3. $(\alpha \wedge) u\lrcorner+u\lrcorner(\alpha \wedge)=\alpha(u)$, for all $\alpha \in V^{*}$ and all $u \in V$.

Proof. Items 1 and 2 are clear. Item 3 holds because for all $u \in V, u\lrcorner: \bigwedge^{k} V^{*} \rightarrow$ $\bigwedge^{k-1} V^{*}$ is an antiderivation of degree -1 : for all $\alpha \in \wedge^{k} V^{*}$ and all $\left.\beta \in \wedge V^{*}, u\right\lrcorner(\alpha \wedge$ $\left.\beta)=(u\lrcorner \alpha) \wedge \beta+(-1)^{k} \alpha \wedge(u\lrcorner \beta\right)$.

Lemma 3.4. Let $n \in \mathbb{N}, \alpha_{1}, \ldots, \alpha_{n} \in V^{*}$ and $v_{1}, \ldots, v_{n} \in V$. Then

$$
\left.\left.\operatorname{det}\left(\alpha_{i}\left(v_{j}\right)\right)_{1 \leq i, j \leq n}=(-1)^{\frac{n(n-1)}{2}} v_{1}\right\lrcorner \ldots v_{n}\right\lrcorner\left(\alpha_{1} \wedge \ldots \wedge \alpha_{n}\right) .
$$

Proof. Observe that (32) makes sense because the right hand side of this equation is an element of $\bigwedge^{0} V^{*}=\mathbb{C}$. Equation (30) implies by induction that

$$
\left.\left.v_{1}\right\lrcorner \ldots v_{n}\right\lrcorner\left(\alpha_{1} \wedge \ldots \wedge \alpha_{n}\right)=\left\langle\alpha_{1} \wedge \ldots \wedge \alpha_{n}, v_{n} \wedge \ldots \wedge v_{1}\right\rangle
$$

Inverting the order of the $v_{i}$ and using (28) gives the claim.

Definition 3.5. Let $(\mathcal{J}, \succ)$ be a totally ordered set. For $j, j^{\prime} \in \mathcal{J}, j \neq j^{\prime}$ denote

$$
1_{j \succ j^{\prime}}= \begin{cases}1 & \text { if } j \succ j^{\prime} \\ 0 & \text { if } j^{\prime} \succ j .\end{cases}
$$

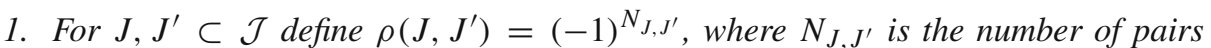
$\left(j, j^{\prime}\right) \in J \times J^{\prime}$ with $j \succ j^{\prime}$.

2. Let $K \in \mathbb{N}$ and $\mathbb{J}=\left(j_{1}, \ldots, j_{K}\right)$ be a finite sequence in $\mathcal{J}$, such that $k \neq l \Rightarrow$ $j_{k} \neq j_{l}$. Let $\pi \in S_{K}$ denote the unique permutation chosen such that for all $k \in$ $\{1, \ldots, K-1\}, j_{\pi(k)} \prec j_{\pi(k+1)}$. Let $\varepsilon_{1}, \ldots, \varepsilon_{K} \in$ End $\bigwedge V^{*}$. The $\mathbb{J}$-chronological product of $\varepsilon_{1}, \ldots, \varepsilon_{K}$ is

$$
\mathbb{T}_{\mathbb{J}}\left[\varepsilon_{1}, \ldots, \varepsilon_{K}\right]=\operatorname{sgn}(\pi) \prod_{\nu=1}^{K} \varepsilon_{\pi(v)} .
$$

3. Let $J=\left\{j_{1}, \ldots, j_{n}\right\}, J^{\prime}=\left\{j_{1}^{\prime}, \ldots, j_{n}^{\prime}\right\}$ with $j_{1} \prec \ldots \prec j_{n}, j_{1}^{\prime} \prec \ldots \prec j_{n}^{\prime}$ and $J \cap J^{\prime}=\emptyset$. Let $\varepsilon_{1}, \ldots, \varepsilon_{2 n} \in$ End $\bigwedge V^{*}$ and $\mathbb{J}=\left(j_{1}, \ldots, j_{n}, j_{1}^{\prime}, \ldots, j_{n}^{\prime}\right)$. For this 
special choice we denote

$$
\mathbb{T}_{J, J^{\prime}}\left[\varepsilon_{1}, \ldots, \varepsilon_{2 n}\right]=\mathbb{T}_{\mathbb{J}}\left[\varepsilon_{1}, \ldots, \varepsilon_{2 n}\right]
$$

and call it the $\left(J, J^{\prime}\right)$-chronological product of $\varepsilon_{1}, \ldots, \varepsilon_{2 n}$.

An obvious consequence is

Lemma 3.6. Let $J$ and $J^{\prime}$ be chosen as in item 3 and $\pi$ as in item 2 of Definition 3.5. Then

$$
\operatorname{sgn}(\pi)=\rho\left(J, J^{\prime}\right) .
$$

This sign is chosen in the definition (35) of the chronological product because in our application the $\varepsilon_{i}$ will be odd elements of the graded algebra End $\wedge V^{*}$. In general, the sign involved in the chronological product is well-defined only if each $\varepsilon_{i}$ is either even or odd, and the sign includes only the permutations of odd elements.

The main result of this section is the following generalization of Lemma 3.4.

Theorem 3.7. Let $(\mathcal{J}, \succ)$ be a totally ordered set and $J$ and $J^{\prime}$ be chosen as in Definition 3.5. For $\alpha_{1}, \ldots, \alpha_{n} \in V^{*}$ and $v_{1}, \ldots, v_{n} \in V$ define the $(n \times n)$-matrix $M$ by

$$
M_{k l}=\alpha_{k}\left(v_{l}\right) 1_{j_{k}^{\prime} \succ j_{l}}
$$

Then

$$
\left.\left.\operatorname{det} M=(-1)^{n(n-1) / 2} \mathbb{T}_{J, J^{\prime}}\left[v_{1}\right\lrcorner, \ldots, v_{n}\right\lrcorner,\left(\alpha_{1} \wedge\right), \ldots,\left(\alpha_{n} \wedge\right)\right] 1 .
$$

Proof. Induction on $n$. The case $n=1$ is obvious. Let $n \geq 2$ and assume (39) to hold for matrices of size $n-1$. By definition and by Lemma 3.6, the chronological product $\mathbb{T}_{J, J^{\prime}}[\cdots]$ on the right-hand side of (38) is $\rho\left(J, J^{\prime}\right) A_{1} \ldots A_{2 n}$, with $A_{i} \in$ $\left.\left\{v_{1}\right\lrcorner, \ldots,\left(\alpha_{n} \wedge\right)\right\}$. Suppose that $A_{1}=\left(\alpha_{m} \wedge\right)$ for some $m$. Then $A_{2} \ldots A_{2 n} 1=0$, so the right hand side of (39) vanishes. The indicator function in the definition of $M$ implies that the $m^{\text {th }}$ row of $M$ is zero, so that the left-hand side of (39) vanishes, too. Thus we may assume that $\left.\left.A_{1} \in\left\{v_{1}\right\lrcorner, \ldots, v_{n}\right\lrcorner\right\}$. Because $J$ is ordered, $\left.A_{1}=v_{1}\right\lrcorner$. Use

$$
\begin{aligned}
A_{1} A_{2} \ldots A_{2 n}= & \sum_{k=2}^{2 n}(-1)^{k} A_{2} \ldots A_{k-1}\left(A_{1} A_{k}+A_{k} A_{1}\right) A_{k+1} \ldots A_{2 n} \\
& -A_{2} \ldots A_{2 n} A_{1} .
\end{aligned}
$$

When applied to $1 \in \bigwedge^{0} V^{*}$, the last term vanishes because $A_{1} 1=0$. By Lemma 3.3, $A_{1} A_{k}+A_{k} A_{1}=\alpha_{m}\left(v_{1}\right)$ if $A_{k}=\alpha_{m} \wedge$ for some $m \in\{1, \ldots, n\}$, and zero otherwise. The position $k$ where $\alpha_{m} \wedge$ appears in the product is

$$
k=1+\left|\left\{j \in J \cup J^{\prime}: j \prec j_{m}^{\prime}\right\}\right|=1+m-1+\left|\left\{j \in J: j \prec j_{m}^{\prime}\right\}\right| .
$$

Thus $(-1)^{k}=(-1)^{m} \rho\left(\left\{j_{m}^{\prime}\right\}, J\right)$. Let $I=J \backslash\{1\}$ and $I_{m}^{\prime}=J^{\prime} \backslash\left\{j_{m}^{\prime}\right\}$. The remaining product $A_{2} \ldots A_{k-1} A_{k+1} \ldots A_{2 n}$ times the sign factor $\rho\left(I, I_{m}^{\prime}\right)$ equals the $\left(I, I_{m}^{\prime}\right)$-chronological product, so

$$
\begin{aligned}
& \left.\left.\mathbb{T}_{J, J^{\prime}}\left[v_{1}\right\lrcorner, \ldots, v_{n}\right\lrcorner,\left(\alpha_{1} \wedge\right), \ldots,\left(\alpha_{n} \wedge\right)\right] 1 \\
& \left.\left.\quad=\sum_{m=1}^{n} \sigma_{m}\left(J, J^{\prime}\right) \mathbb{T}_{I, I_{m}^{\prime}}\left[v_{2}\right\lrcorner, \ldots, v_{n}\right\lrcorner,\left(\alpha_{1} \wedge\right), \ldots,\left(\alpha_{m-1} \wedge\right),\left(\alpha_{m+1} \wedge\right), \ldots,\left(\alpha_{n} \wedge\right)\right] 1
\end{aligned}
$$


with

$$
\sigma_{m}\left(J, J^{\prime}\right)=\rho\left(J, J^{\prime}\right)(-1)^{m} \rho\left(\left\{j_{m}^{\prime}\right\}, J\right) \rho\left(I, I_{m}^{\prime}\right) .
$$

By definition,

$$
\rho\left(J, J^{\prime}\right)=\rho\left(I, I_{m}^{\prime}\right) \rho\left(J,\left\{j_{m}^{\prime}\right\}\right) \rho\left(\{1\}, I_{m}^{\prime}\right),
$$

$\rho\left(\{1\}, I_{m}^{\prime}\right)=1$, and

$$
\rho\left(\left\{j_{m}^{\prime}\right\}, J\right) \rho\left(J,\left\{j_{m}^{\prime}\right\}\right)=(-1)^{|J|}=(-1)^{n} .
$$

Thus

$$
\sigma_{m}\left(J, J^{\prime}\right)=(-1)^{m+n} .
$$

The inductive hypothesis applies to the chronological product on the right hand side of (42). Combine $(-1)^{n(n-1) / 2+m+n}=(-1)^{(n-1)(n-2) / 2}(-1)^{m-1}$. The statement of the theorem follows by identifying the right hand side of (42) as the Laplace expansion for the determinant.

In the remainder of this section, we prepare and give the proof of Theorem 1.3.

Lemma 3.8. Assume that the space $V$ is a Hilbert space with scalar product $\langle\cdot, \cdot\rangle_{V}$. In this case we identify $V$ with its dual $V^{*}\left(v \in V \mapsto\langle v, \cdot\rangle_{V} \in V^{*}\right)$ and consequently $\bigwedge^{k} V$ with $\bigwedge^{k} V^{*} \cong\left(\bigwedge^{k} V\right)^{*}$ (see (28) and (29)).

1. The scalar product $\langle\cdot, \cdot\rangle_{V}$ of $V$ induces, for each $k \in \mathbb{N}$, through the identification of elements of $\bigwedge^{k} V$ with elements of its dual $\left(\bigwedge^{k} V\right)^{*}$ a norm $\|\cdot\|$ on $\bigwedge^{k} V$ : $\|u\|^{2}=\langle u, u\rangle$. This norm fulfills the parallelogram identity

$$
\|u+v\|^{2}+\|u-v\|^{2}=2\|u\|^{2}+2\|v\|^{2}, \quad \forall u, v \in \bigwedge^{k} V,
$$

hence it defines a compatible scalar product on $\wedge^{k} V$. Thus $\wedge^{k} V$ and hence $\bigwedge V$ are Hilbert spaces.

2. $(u\lrcorner)^{\dagger}=(u \wedge)$ and $\left.(u \wedge)^{\dagger}=u\right\lrcorner$, for all $u \in V$.

3. $\max \{\| u\lrcorner\|\|,(u \wedge) \|\} \leq\|u\|$, for all $u \in V$.

Proof. 1. To see that $\|\cdot\|$ is nondegenerate, use the defining identity (28). The other properties are clear. Item 2 follows directly from Definition 3.2.1. To see 3, let $u \in V$ and $w \in \bigwedge V$. Then by Lemma 3.3,

$$
\langle w,(u\lrcorner(u \wedge)+(u \wedge) u\lrcorner) w\rangle=\|w\|^{2}\|u\|^{2} .
$$

Thus $\left.\left.\left.\|u\|^{2}=\sup _{\substack{w \in \wedge V \\\|w\|=1}}\langle w,(u\lrcorner(u \wedge)+(u \wedge) u\lrcorner\right) w\right\rangle \geq \max \{\| u\lrcorner\left\|^{2},\right\|(u \wedge) \|^{2}\right\}$.

In Definition 3.1, we required the space $V$ to be finite-dimensional, to avoid a discussion of subtleties in the relation between $\bigwedge V$ and its dual. In our applications, we can always achieve that $V$ is a finite-dimensional subspace of a Hilbert space or a reflexive Banach space, by taking $V$ as a space spanned by finitely many vectors. For Hilbert spaces, we could alternatively also have dropped the condition of finite dimensionality in the above. 
Lemma 3.9. Let $\varphi, \varphi^{\prime}: \mathbb{N} \rightarrow \mathcal{J}$ be functions into a totally ordered $\operatorname{set}(\mathcal{J}, \succ)$. Let $\mathcal{H}$ be a Hilbert space. For all $n \in \mathbb{N}$ and all $v_{1}, \ldots, v_{n}, w_{1}, \ldots, w_{n} \in \mathcal{H}$,

$$
\left|\operatorname{det}\left(\left\langle v_{k}, w_{l}\right\rangle_{\mathcal{H}} 1_{\varphi^{\prime}(k) \succ \varphi(l)}\right)_{k, l}\right| \leq \prod_{k=1}^{n}\left\|v_{k}\right\|\left\|w_{k}\right\| .
$$

The same inequality holds with $1_{\varphi^{\prime}(k) \succ \varphi(l)}$ replaced by $1_{\varphi^{\prime}(k) \succeq \varphi(l)}$.

Proof. For $n \geq 1$ let $\mathbb{N}_{n}=\{1, \ldots, n\}$. Define

$$
\mathcal{G}_{n}=\left\{j \in \mathcal{J}: \exists k, l \in \mathbb{N}_{n}: \varphi^{\prime}(k)=\varphi(l)=j\right\} .
$$

Obviously, $\left|\mathcal{G}_{n}\right| \leq n$. Let

$$
m=\max _{j \in \mathcal{J}}\left\{\left(\varphi^{\prime}\right)^{-1}(\{j\}) \cap \mathbb{N}_{n}, \varphi^{-1}(\{j\}) \cap \mathbb{N}_{n}\right\}
$$

and set $\tilde{\mathcal{J}}_{n}=\mathcal{J} \times\{0,1\} \times\{1, \ldots, m\}$. Extend the ordering lexicographically, i.e. $(j, \mu, v) \succ\left(j^{\prime}, \mu^{\prime}, v^{\prime}\right) \Leftrightarrow j \succ j^{\prime}$ or $\left[j=j^{\prime}\right.$ and $\left.\mu \succ \mu^{\prime}\right]$ or $\left[j=j^{\prime}\right.$ and $\mu=\mu^{\prime}$ and $v>v^{\prime}$ ]. Then $\left(\tilde{\mathcal{J}}_{n}, \succ\right)$ is totally ordered. For $j \in \mathcal{G}_{n}$, there are $r \leq m$ and $k_{1}, \ldots, k_{r} \in$ $\mathbb{N}_{n}$ such that for all $\rho \leq r, \varphi^{\prime}\left(k_{\rho}\right)=j$, and there are $s \leq m, l_{1}, \ldots, l_{s} \in \mathbb{N}_{n}$ such that for all $\sigma \leq s, \varphi\left(l_{\sigma}\right)=j$. We now extend $\varphi$ to $\tilde{\varphi}$ and $\varphi^{\prime}$ to $\tilde{\varphi}^{\prime}$ as follows.

Case of the matrix with $1_{\varphi^{\prime}(k) \succ \varphi(l)}$. In this case, $1_{\varphi^{\prime}(k) \succ \varphi(l)}=0$ if $\varphi^{\prime}(k)=\varphi(l)$. To obtain $1_{\tilde{\varphi}^{\prime}(k) \succ \tilde{\varphi}(l)}=0$, we make $\tilde{\varphi}^{\prime}(k)$ smaller by setting $\tilde{\varphi}^{\prime}\left(k_{\rho}\right)=\left(\varphi^{\prime}\left(k_{\rho}\right), 0, \rho\right)$ and $\tilde{\varphi}\left(l_{\sigma}\right)=\left(\varphi\left(l_{\sigma}\right), 1, \sigma\right)$.

Case of the matrix with $1_{\varphi^{\prime}(k) \succeq \varphi(l)}$. In this case, $1_{\varphi^{\prime}(k) \succeq \varphi(l)}=1$ if $\varphi^{\prime}(k)=\varphi(l)$. To obtain $1_{\tilde{\varphi}^{\prime}(k) \succ \tilde{\varphi}(l)}=1$, we make $\tilde{\varphi}^{\prime}(k)$ bigger by setting $\tilde{\varphi}^{\prime}\left(k_{\rho}\right)=\left(\varphi^{\prime}\left(k_{\rho}\right), 1, \rho\right)$ and $\tilde{\varphi}\left(l_{\sigma}\right)=\left(\varphi\left(l_{\sigma}\right), 0, \sigma\right)$.

For $j \in \mathcal{J} \backslash \mathcal{G}_{n}, j=\varphi^{\prime}(k)$, we set $\tilde{\varphi}^{\prime}(k)=\left(\varphi^{\prime}(k), 0, \rho\right)$ etc. By definition of the lexicographical ordering on $\tilde{\mathcal{J}}$, it does not matter which convention one chooses on $\mathcal{J} \backslash \mathcal{G}_{n}$.

By construction, $\tilde{\varphi}^{\prime}\left(\mathbb{N}_{n}\right)=J^{\prime}$ and $\tilde{\varphi}\left(\mathbb{N}_{n}\right)=J$ are disjoint, and $|J|=\left|J^{\prime}\right|=n$. We may permute the rows and columns of the matrix such that $\tilde{\varphi}\left(m_{1}\right) \prec \tilde{\varphi}\left(m_{2}\right)$ if $m_{1}<m_{2}$ and similarly for $\tilde{\varphi}^{\prime}$. This does not change the absolute value of the determinant. We can now apply Theorem 3.7, to represent the determinant as a chronological product. The norm estimate in Lemma 3.8 implies the statement.

Definition 3.10. Let $n \in \mathbb{N}$ and $A$ be a complex $(n \times n)$-matrix. We say that $\Pi(A, \gamma)$ holds iff for all $p \in\{1, \ldots n\}$ and all sequences $a_{1}<\cdots<a_{p}$ and $b_{1}<\cdots<b_{p}$ in $\{1, \ldots, n\}$,

$$
\sup _{v_{1}, \ldots, v_{p}, w_{1}, \ldots, w_{p} \in B_{1}^{(n)}}\left|\operatorname{det}\left(\left\langle v_{q}, w_{r}\right\rangle A_{a_{q}, b_{r}}\right)_{1 \leq q, r \leq p}\right| \leq \gamma^{2 p}
$$

Lemma 3.11. Let $n$ and $k \in \mathbb{N}$ and $A^{(1)}, \ldots, A^{(k)}$ be complex $(n \times n)$-matrices. Assume that for all $l \in\{1, \ldots, k\}$ there are $\gamma_{l}>0$ such that the property $\Pi\left(A^{(l)}, \gamma_{l}\right)$ holds. Then $\Pi\left(A^{(1)}+\cdots+A^{(k)}, \gamma_{1}+\cdots+\gamma_{k}\right)$ holds. 
Proof. Induction on $k$. For $k=1$, the statement is obvious. In the induction step, let $k \geq$ 2 , and assume $\Pi\left(A^{(2)}+\cdots+A^{(k)}, \gamma_{2}+\cdots+\gamma_{k}\right)$ to hold. Let $p \in\{1, \ldots n\}, a_{1}<\cdots<$ $a_{p}$, and $b_{1}<\ldots<b_{p}$ in $\{1, \ldots, n\}$, and $v_{1}, \ldots, v_{p}, w_{1}, \ldots, w_{p} \in B_{1}^{(n)}$. Let $B$ and $C$ be the matrices with elements $B_{q, r}=\left\langle v_{q}, w_{r}\right\rangle A_{a_{q}, b_{r}}^{(1)}$ and $C_{q, r}=\left\langle v_{q}, w_{r}\right\rangle \sum_{i=2}^{k} A_{a_{q}, b_{r}}^{(i)}$ Also, set $\gamma_{1}^{\prime}=\sum_{l=2}^{k} \gamma_{l}$. Then by the generalized Laplace expansion for determinants

$$
\operatorname{det}(B+C)=\sum_{\substack{S, T \subset\{1, \ldots, p\} \\|S|=|T|}} \varepsilon_{p}(S, T) \operatorname{det} B_{S, T} \operatorname{det} C_{S^{c}, T^{c}},
$$

where $S^{c}=\{1, \ldots, p\} \backslash S$ and $\varepsilon_{p}(S, T) \in\{-1,1\}$, and the subscripts denote the submatrices of $B$ and $C$ defined by the sets. Let $s=|S|=|T|$. By hypothesis of the lemma, for all $S, T$,

$$
\left|\operatorname{det} B_{S, T}\right| \leq \gamma_{1}^{2 s}
$$

and by the inductive hypothesis,

$$
\left|\operatorname{det} C_{S^{c}, T^{c}}\right| \leq \gamma_{1}^{\prime 2(p-s)}
$$

Thus, using $\left(\begin{array}{l}p \\ s\end{array}\right)^{2} \leq\left(\begin{array}{c}2 p \\ 2 s\end{array}\right)$

$$
|\operatorname{det}(B+C)| \leq \sum_{s=0}^{p}\left(\begin{array}{l}
p \\
s
\end{array}\right)^{2} \gamma_{1}{ }^{2 s} \gamma_{1}^{\prime 2(p-s)} \leq\left(\sum_{l=1}^{k} \gamma_{l}\right)^{2 p} .
$$

Proof of Theorem 1.3.. Call the $n \times n$ submatrices of the summands in (8) $M_{l}$. By Lemma 3.11, it suffices to show that for all $l \in\{0, \ldots, k+K\}, \Pi\left(M_{l}, \gamma_{l}\right)$ holds. The matrix $C_{l}$ has a Gram representation $(\mathcal{H}, g, h)$ with Gram constant $\gamma_{l}$. Then

$$
\langle v, w\rangle_{\mathbb{C}^{n}}\left(C_{l}\right)_{x y}=\left\langle v \otimes g_{x}, w \otimes h_{y}\right\rangle_{\mathbb{C}^{n} \otimes \mathcal{H}}
$$

and, if $\|v\| \leq 1,\left\|v \otimes g_{x}\right\|=\|v\|\left\|g_{x}\right\| \leq\left\|g_{x}\right\|$, similarly for $w \otimes h_{y} . M_{l}$ is obtained (for $l>0$ ) by multiplying this with an indicator function. Every submatrix of $M_{l}$ is of the same form as $M_{l}$ and satisfies the hypotheses of Lemma 3.9. Thus $\Pi\left(M_{l}, \gamma_{l}\right)$ holds.

That all submatrices are involved in property $\Pi$, as necessary for the inductive argument in the proof of Lemma 3.11, is the reason for taking the supremum over the larger set in Definition 1.2, instead of taking a supremum over $P \in \mathcal{P}_{n, 1}$. Submatrices of a $P \in \mathcal{P}_{n, 1}$ are in general not positive. By contrast, the property of having a Gram representation on $\mathbb{C}^{n}$ with Gram constant 1 is stable under taking submatrices.

\section{Convergent Expansions without UV Cutoffs}

In this section we apply the results of Sect. 3 to the many-fermion covariances introduced in Sect. 2. We give explicit determinant and decay bounds, and prove Theorem 2.4. Moreover, we show that, for a multiscale expansion with the standard Fermi surface cutoff functions and sectorization, our results yield all standard power counting bounds without requiring a cutoff on the Matsubara frequencies, so that the analytic structure as a function of the frequencies can be preserved in such a multiscale analysis. 
4.1. Determinant bound. In the following, we apply Theorem 1.3 to the covariance (14), of which (13) is the special case $\mathbf{h}=1$. Before stating the details of the representation we briefly motivate it. By definition,

$$
\mathbb{C}(\tau, E)=-1_{\tau>0} \mathrm{e}^{-\tau E} f_{\beta}(-E)+1_{\tau \leq 0} \mathrm{e}^{-\tau E} f_{\beta}(E)
$$

Let $\varepsilon>0$ and

$$
\Phi(s, \varepsilon)=\frac{1}{\sqrt{\pi}} \frac{\sqrt{\varepsilon f_{\beta}(-\varepsilon)}}{\mathrm{i} s-\varepsilon} .
$$

Then, since $\varepsilon>0, s \mapsto \Phi(s, \varepsilon) \in L^{2}(\mathbb{R}),\|\Phi(\cdot, \varepsilon)\|_{2} \leq 1$, and,

$$
\forall \tau \geq 0, \varepsilon>0: \quad \mathrm{e}^{-\varepsilon \tau} f_{\beta}(-\varepsilon)=\int_{\mathbb{R}} \mathrm{d} s \mathrm{e}^{\mathrm{i} s \tau}|\Phi(s, \varepsilon)|^{2}
$$

Thus, if $\tau=t-t^{\prime}>0, \mathrm{e}^{-\varepsilon \tau} f_{\beta}(-\varepsilon)=\left\langle v_{t}, v_{t^{\prime}}\right\rangle$ with $v_{t}(s)=\mathrm{e}^{-i s t} \Phi(s, \varepsilon)$. To use this for $\mathbb{C}$ we need to respect the signs in (58), hence rewrite, for $\tau \in[-\beta, \beta]$,

$$
\mathbb{C}(\tau, E)= \begin{cases}-\mathrm{e}^{-\tau E} f_{\beta}(-E) & \text { if } \tau>0 \text { and } E>0 \\ -\mathrm{e}^{(\beta-\tau) E} f_{\beta}(E) & \text { if } \tau>0 \text { and } E<0 \\ \mathrm{e}^{-(\beta+\tau) E} f_{\beta}(-E) & \text { if } \tau \leq 0 \text { and } E>0 \\ \mathrm{e}^{-\tau E} f_{\beta}(E) & \text { if } \tau \leq 0 \text { and } E<0\end{cases}
$$

using $f_{\beta}(-E)=\mathrm{e}^{\beta E} f_{\beta}(E)$. By Tonelli's theorem and an obvious decomposition of the remaining factors in the integrand, we can represent $C_{(t, x),\left(t^{\prime}, x^{\prime}\right)}$ by integration over $\mathrm{p}$. Note that the $v_{t}$ defined above vanishes at $E=0$, but that $\mathbb{C}(\tau, 0)=\frac{1}{2}-1_{\tau>0} \neq 0$, so it is necessary to restrict to functions $E(\mathrm{p})$ whose zero level set has measure zero.

Lemma 4.1. Let $E: \mathcal{B} \rightarrow \mathbb{R}$ be measurable and assume that

$$
\mu^{a}(\{\mathrm{p} \in \mathcal{B}: E(\mathrm{p})=0\})=0
$$

Let $\mathbf{h} \in L^{1}\left(\mathcal{B}, \mu^{a}\right)$ with $\mathbf{h}(\mathrm{p}) \geq 0$ for all $\mathrm{p} \in \mathcal{B}$. For $x=(t, \mathrm{x}) \in \mathbb{X}_{d}$ and $\sigma \in\{-1,1\}$ define

$$
\begin{aligned}
& g_{x}^{\sigma}(s, \mathrm{p})=\mathrm{e}^{-\mathrm{i} \cdot \mathrm{x}-\mathrm{i} s t} \Phi(s,|E(\mathrm{p})|) \sqrt{\mathbf{h}(\mathrm{p})} 1_{\sigma E(\mathrm{p})>0}, \\
& h_{x}(s, \mathrm{p})=\mathrm{e}^{-\mathrm{ip} \cdot \mathrm{x}+\mathrm{i} s t} \Phi(s,|E(\mathrm{p})|) \sqrt{\mathbf{h}(\mathrm{p})} 1_{E(\mathrm{p})<0} .
\end{aligned}
$$

Then for all $x \in \mathbb{X}_{d}, g_{x}^{+}, g_{x}^{-}$and $h_{x}$ are in $\mathcal{H}=L^{2}\left(\mathbb{R} \times \mathcal{B}, \mathrm{d} s \otimes \mathrm{d} \mu^{a}\right)$, with norms bounded by $\|\mathbf{h}\|_{1}^{1 / 2}$, and the covariance (14) has the representation

$$
\begin{aligned}
C_{(t, x),\left(t^{\prime}, x^{\prime}\right)}^{(\mathbf{h})}= & 1_{t>t^{\prime}}\left\langle-g_{t, \mathrm{x}}^{+}-g_{\beta-t, \mathrm{x}}^{-}, g_{t^{\prime}, \mathrm{x}^{\prime}}^{+}+h_{t^{\prime}, \mathrm{x}^{\prime}}\right\rangle \\
& +1_{t \leq t^{\prime}}\left\langle g_{t, \mathrm{x}}^{+}+h_{t, \mathrm{x}}, g_{t^{\prime}-\beta, \mathrm{x}^{\prime}}^{+}+h_{t^{\prime}, \mathrm{x}^{\prime}}\right\rangle .
\end{aligned}
$$


Proof. The integrand in (14) is bounded, so we can remove the set of measure zero $\{\mathrm{p} \in \mathcal{B}: E(\mathrm{p})=0\}$ from the integral. On its complement, the Gram representation given in the lemma converges absolutely as an iterated integral first over $s$, then over $\mathrm{p}$, hence by Tonelli's theorem in any order of integration, and the $L^{2}$-norms are finite by the same argument. The bound for the norms is obvious from the properties of $\Phi$. By the support properties of the functions,

$$
\left\langle-g_{t, \mathrm{x}}^{+}-g_{\beta-t, \mathrm{x}}^{-}, g_{t^{\prime}, \mathrm{x}^{\prime}}^{+}+h_{t^{\prime}, \mathrm{x}^{\prime}}\right\rangle=\left\langle-g_{t, \mathrm{x}}^{+}, g_{t^{\prime}, \mathrm{x}^{\prime}}^{+}\right\rangle+\left\langle-g_{\beta-t, \mathrm{x}}^{-}, h_{t^{\prime}, \mathrm{x}^{\prime}}\right\rangle
$$

and

$$
\left\langle g_{t, \mathrm{x}}^{+}+h_{t, \mathrm{x}}, g_{t^{\prime}-\beta, \mathrm{x}^{\prime}}^{+}+h_{t^{\prime}, \mathrm{x}^{\prime}}\right\rangle=\left\langle g_{t, \mathrm{x}}^{+}, g_{t^{\prime}-\beta, \mathrm{x}^{\prime}}^{+}\right\rangle+\left\langle h_{t, \mathrm{x}}, h_{t^{\prime}, \mathrm{x}^{\prime}}\right\rangle .
$$

Decomposing the integration domain into $\mathcal{B}_{ \pm}=\{\mathrm{p} \in \mathcal{B}: \pm E(\mathrm{p})>0\},(64)$ follows from (60) and (61).

The condition that $\mathbf{h} \geq 0$ in Lemma 4.1 was just for convenience in stating the result in a simple form. With an obvious generalization, replacing $\sqrt{\mathbf{h}(p)}$ by $\mathbf{h}(\mathrm{p})|\mathbf{h}(\mathrm{p})|^{-1 / 2}$, and defining a few more functions $\tilde{g}$ to take care of the necessary complex conjugations, a representation with the same properties as (64) can be obtained for general $\mathbf{h} \in L^{1}\left(\mathcal{B}, \mu^{a}\right)$. In the applications below, $\mathbf{h}$ will be a scaling function, hence nonnegative.

Corollary 4.2. Under the hypotheses of Lemma 4.1, the many-fermion covariance (14) has a determinant bound $\delta_{C}(\mathbf{h})$ with

$$
\frac{1}{\sqrt{2}}\|\mathbf{h}\|_{1}^{1 / 2} \leq \delta_{C^{(\mathbf{h})}} \leq 2\|\mathbf{h}\|_{1}^{1 / 2}
$$

(for $\mathbf{h}=1$, corresponding to the covariance (13), $\left.\|\mathbf{h}\|_{1}=\mu^{a}(\mathcal{B})=a^{-d}\right)$.

Proof. The indicator functions in the times $t$ and $t^{\prime}$ correspond to the choices $(\mathcal{J}, \succ)=$ $([-\beta, \beta],>), \varphi_{1}(t, \mathrm{x})=\varphi_{1}^{\prime}(t, \mathrm{x})=t$ and $\varphi_{2}(t, \mathrm{x})=\varphi_{2}^{\prime}(t, \mathrm{x})=-t$. The upper bound follows from the explicit representation given in Lemma 4.1 by applying Theorem 1.3. Let

$$
\rho_{ \pm}=\int_{\mathcal{B}} \mu^{a}(\mathrm{dp}) f_{\beta}( \pm E(\mathrm{p})) \mathbf{h}(\mathrm{p}),
$$

then $\rho_{-}=\|\mathbf{h}\|_{1}-\rho_{+}$. Set $\mathrm{x}=\mathrm{x}^{\prime}$. Then considering the cases $t=t^{\prime}$ and $t^{\prime} \uparrow t$ gives

$$
\sup _{x, x^{\prime} \in \mathbb{X}_{d}}\left|C_{x x^{\prime}}^{(\mathbf{h})}\right| \geq \max \left\{\rho_{+}, \rho_{-}\right\} \geq \frac{1}{2}\|\mathbf{h}\|_{1} .
$$

The lower bound for $\delta_{C^{(\mathbf{h})}}$ now follows from (10).

Proof of Theorem 2.4. To apply Lemma 4.1, we need to satisfy the zero measure condition. For $\varepsilon>0$, define $E_{\varepsilon}: \mathcal{B} \rightarrow \mathbb{R}$ by $E_{\varepsilon}(\mathrm{p})=\varepsilon / 2$ if $|E(\mathrm{p})| \leq \varepsilon / 2$ and $E_{\varepsilon}(\mathrm{p})=E(\mathrm{p})$ otherwise. Obviously, $\left\|E-E_{\varepsilon}\right\|_{\infty} \leq \varepsilon$, and $\left\{\mathrm{p} \in \mathcal{B}: E_{\varepsilon}(\mathrm{p})=0\right\}=\varnothing$. Because $\beta<\infty$, the covariance $C^{(\mathbf{h})}$ is a continuous function of $E$ in $\|\cdot\|_{\infty}$, so $C^{(\mathbf{h})}$ is the limit $\varepsilon \rightarrow 0$ of the covariance $C^{(\mathbf{h}, \varepsilon)}$ given by $E_{\varepsilon}$. By construction, $E_{\varepsilon}$ satisfies the conditions of Lemma 4.1 so Corollary 4.2 implies the bound (25) for $C^{(\mathbf{h}, \varepsilon)}$. That bound is uniform in $\varepsilon$. 
The representation of $C^{(\mathbf{h})}$ given in Lemma 4.1 generalizes one found in [FKT04], where determinants of matrices of the form

$$
M_{k l}=\left\langle v_{k}, w_{l}\right\rangle\left\{\begin{array}{cc}
0, & t_{k}-t_{l} \leq 0 \\
e^{-\left(t_{k}-t_{l}\right)}, & t_{k}-t_{l}>0
\end{array},\right.
$$

for vectors $v_{k}, w_{l}$ in a Hilbert space $\mathcal{H}$ and real numbers $t_{k}, t_{l}$, were considered. The result of [FKT04] corresponds to the special case of the function

$$
\tilde{\mathbb{C}}(\tau)=-\mathrm{e}^{-\tau} 1_{\tau>0},
$$

which is the limit $\beta \rightarrow \infty$ of (58) at $E=1$. Thus our method applies to that case, with $\tilde{\Phi}(s)=(\text { is }-1)^{-1}$.

4.2. Decay constant. Under very mild conditions on $E$, the determinant bounds we have proven are uniform in $\beta$ (see Corollary 4.2). One must of course not jump to the conclusion that this implies convergence of perturbation series uniformly in the temperature because a finite determinant bound is only one condition for convergence of the perturbation expansion. The second is the finiteness of the decay constants

$$
\alpha_{C}^{\left(k_{0}, k\right)}=\int_{-\beta}^{\beta} \mathrm{d} \tau \int_{X} \mathrm{dx}|C(\tau, \mathrm{x})||\tau|^{k_{0}}|x|^{k}
$$

for $k_{0} \geq 0$ and $k \geq 0$. In this paper, we only discuss the case $k_{0}=k=0$, and denote $\alpha_{C}^{(0,0)}=\alpha_{C}$ because the simplest convergence theorem requires only this data, and because the generalization is straightforward. For our many-fermion covariance, the existence of a nonempty Fermi surface that is not degenerated to a point implies that the decay constant grows polynomially in $\beta$ and diverges in the zero-temperature limit. Only for special situations, such as a model for an insulator, for which $|E(\mathrm{p})| \geq E_{\min }>0$, the decay constant is uniform in $\beta$.

For simplicity we assume here the case of a continuous torus $\mathcal{B}$. The case of a discrete torus corresponding to a finite volume is similar, and treated in [PS].

For $z \in \mathbb{C}$ and $\varepsilon \geq 0$ set $\|z\|_{\varepsilon}=\max \{|z|, \varepsilon\}$.

Lemma 4.3. Let $E \in C^{d+2}(\mathcal{B}, \mathbb{R})$. Let $0<\epsilon<1$ and assume that $\mathbf{h}(\mathrm{p})=f\left(\frac{E(\mathrm{p})}{\epsilon}\right) g(\mathrm{p})$, where $f \in C^{\infty}\left(\mathbb{R}, \mathbb{R}_{0}^{+}\right)$and $g \in C^{\infty}\left(\mathcal{B}, \mathbb{R}_{0}^{+}\right)$. Let $\mathbf{b} \in \mathbb{N}_{0}^{d}$ be a multiindex and $b=|\mathbf{b}|$.

1. There is a constant $K_{d}>0$ such that for $b \leq d+1$,

$$
\int_{-\beta}^{\beta} \mathrm{d} \tau\left|\mathrm{x}^{\mathbf{b}} C_{(\tau, \mathrm{x}),(0,0)}^{(\mathbf{h})}\right| \leq K_{d} \sum_{m=0}^{b} \epsilon^{m-b} \int_{\text {supp } \mathbf{h}} \frac{\mu^{a}(\mathrm{dp})}{\|E(\mathrm{p})\|_{\frac{1}{\beta}}^{m+1}} .
$$

2. If there is $\kappa_{0}>0$ such that for all $E$ for which $\hat{S}_{E, g}=\{\mathrm{p} \in \operatorname{supp} g: E(\mathrm{p})=E\}$ is nonempty, inf ${ }_{\mathrm{p} \in \hat{S}_{E, g}}|\nabla E(\mathrm{p})| \geq \eta>0$, and the submanifold $\hat{S}_{E, g}$ of $\mathcal{B}$ has Gauss curvature bounded below pointwise by $\kappa_{0}$, then there is a constant $\tilde{K}_{d}>0$ such that for $b \leq\left\lceil\frac{d+2}{2}\right\rceil$,

$$
\int_{-\beta}^{\beta} \mathrm{d} \tau\left|\mathrm{x}^{\mathbf{b}} C_{(\tau, \mathrm{x}),(0,0)}^{(\mathbf{h})}\right| \leq \frac{\tilde{K}_{d}}{|\mathrm{x}|^{\frac{d-1}{2}}} \sum_{m=0}^{b} \epsilon^{m-b} \int \mathrm{d} E \frac{1_{\frac{E}{\epsilon} \in \operatorname{supp} f}}{\|E\|_{\frac{1}{\beta}}^{m+1}} .
$$


Proof. We have

$$
\mathrm{x}^{\mathbf{b}} C_{(\tau, \mathrm{x}),(0,0)}^{(\mathbf{h})}=\int \mu^{a}(\mathrm{dp}) \mathbb{C}(\tau, E(\mathrm{p})) \mathbf{h}(\mathrm{p})\left(-\mathrm{i} \frac{\partial}{\partial \mathrm{p}}\right)^{\mathbf{b}} \mathrm{e}^{\mathrm{ip} \cdot \mathrm{x}} .
$$

Upon integration by parts, the derivative can act in four places - on $\mathbb{C}$, on either of the factors $f$ and $g$ in $\mathbf{h}$, or (for the continuum system) on the spatial ultraviolet cutoff function $\chi$ in $\mu^{a}(\mathrm{dp})=\chi(a \mathrm{p}) \mathrm{dp}$. Thus

$$
\mathrm{x}^{\mathbf{b}} C_{(\tau, \mathrm{x}),(0,0)}^{(\mathbf{h})}=\sum_{m=0}^{b} \sum_{n=0}^{b-m} \epsilon^{-n} \int \mu^{a}(\mathrm{dp}) \Gamma_{m}(\tau, E(\mathrm{p})) f^{(n)}\left(\frac{E(\mathrm{p})}{\epsilon}\right) G_{m, n}^{(\mathbf{b})}(\mathrm{p}) \mathrm{e}^{\mathrm{ip} \cdot \mathrm{x}},
$$

where $G_{m, n}^{(\mathbf{b})} \in C^{d+2-b}(\mathcal{B}, \mathbb{R})$ is independent of $\epsilon$ and satisfies supp $G_{m, n}^{(\mathbf{b})} \subset \operatorname{supp} g$, and

$$
\Gamma_{m}(\tau, E)=\frac{\mathrm{d}^{m}}{\mathrm{~d} E^{m}} \mathbb{C}(\tau, E) .
$$

Taking the absolute value inside all sums and integrals and using that

$$
\int_{-\beta}^{\beta} \mathrm{d} \tau\left|\Gamma_{m}(\tau, E)\right| \leq \text { const }\|E\|_{\frac{1}{\beta}}^{-m-1},
$$

we obtain (72). To prove (73), we rewrite

$$
\mathrm{x}^{\mathbf{b}} C_{(\tau, \mathrm{x}),(0,0)}^{(\mathbf{h})}=\sum_{m=0}^{b} \sum_{n=0}^{b-m} \epsilon^{-n} \int \mathrm{d} E \Gamma_{m}(\tau, E) f^{(n)}\left(\frac{E}{\epsilon}\right) S_{E, G_{m, n}^{(\mathbf{b})}}(\mathrm{x}),
$$

where

$$
S_{E, G_{m, n}^{(\mathbf{b})}}(\mathrm{x})=\int \mu^{a}(\mathrm{dp}) \delta(E-E(\mathrm{p})) G_{m, n}^{(\mathbf{b})}(\mathrm{p}) \mathrm{e}^{\mathrm{ip} \cdot \mathrm{x}} .
$$

By standard theorems about the Fourier transform of surfaces [St],

$$
\left|S_{E, G_{m, n}^{(\mathbf{b})}}(\mathrm{x})\right| \leq \text { const }|\mathrm{x}|^{-\frac{d-1}{2}}
$$

with a constant that depends on $\kappa_{0}$ and $E$, and which is finite under our regularity assumption on $E$. Finally, we use again (76).

The regularity assumptions on $E$ in Lemma 4.3 are not optimized. For improved bounds using smoothing techniques, see [PS]. The scaling function $\mathbf{h}$ can be chosen $C^{\infty}$ in our applications, so that the assumptions of Lemma 4.3 on $\mathbf{h}$ are not restrictive.

This lemma allows us to bound decay constants as follows.

Corollary 4.4. Let $E \in C^{d+2}(\mathcal{B}, \mathbb{R})$.

1. $\alpha_{C} \leq$ const $\beta^{d+1}$.

2. If the system is an insulator, i.e. if there is $E_{0}>0$ such that for all $\mathrm{p} \in \mathcal{B},|E(\mathrm{p})| \geq E_{0}$, then

$$
\alpha_{C^{(\mathbf{h})}} \leq \text { const } E_{0}^{-d-1} .
$$

The constant is proportional to the volume of the support of $\mathbf{h}$. For $\mathbf{h}=1$, it is proportional to $\mu^{a}(\mathcal{B})$. 
If there is $E_{1}$ such that for all energies $E$ with $|E| \leq E_{1}$ the level sets satisfy the hypotheses of Lemma 4.3, item 2, then we also have:

3.

$$
\alpha_{C} \leq \text { const }\left(E_{1}^{-d-1}+\beta^{\frac{d+3}{2}}\right) .
$$

4. If $f(x)=0$ unless $1 \leq|x| \leq 2$, then

$$
\alpha_{C^{(\mathbf{h})}} \leq \text { const } \epsilon^{-\frac{d+1}{2}} .
$$

5. For a sector of angular radius $\sqrt{\epsilon}$, i.e. $g(p)=\gamma\left(\frac{p}{\sqrt{\epsilon}}\right)$, with $\gamma$ supported near 0 , $\alpha_{C^{(h)}} \leq$ const $\epsilon^{-1}$.

Proof. The first bound follows by the standard summation argument from $\|E(\mathrm{p})\|_{\frac{1}{\beta}} \geq$ $\frac{1}{\beta}$. The case of an insulator follows immediately from $\|E(\mathrm{p})\|_{\frac{1}{\beta}} \geq E_{0}$. To prove (81), we insert a partition of unity $\chi_{<}\left(\frac{E(\mathrm{p})}{\epsilon}\right)+\chi_{>}\left(\frac{E(\mathrm{p})}{\epsilon}\right)=1$, where $\chi_{<}(x)$ vanishes for $|x| \geq 1$. The support condition on $f$ in item 4 implies $\|E(\mathrm{p})\|_{\frac{1}{\beta}} \geq \epsilon$. Again, summation implies the result. The sector estimate is similar.

4.3. Convergence theorem. In the following we state a theorem about convergence of expansions for the effective action which generalizes the main theorem of [SW]. As in [SW], we define an interaction $V$ by its interaction vertices $v_{n, m}: \mathbb{X}^{n} \times \mathbb{X}^{m} \rightarrow \mathbb{C}$ as

$$
V(\Psi)=\sum_{m, n \geq 0} \int \mathrm{d}^{n} \underline{X} \mathrm{~d}^{m} \underline{X}^{\prime} v_{n, m}\left(\underline{X}, \underline{X^{\prime}}\right) \bar{\psi}^{n}(\underline{X}) \psi^{m}\left(\underline{X^{\prime}}\right),
$$

where $\underline{X}=\left(X_{1}, \ldots X_{m}\right)$ and $\psi^{m}(\underline{X})=\psi\left(X_{1}\right) \ldots \psi\left(X_{m}\right)$. For $h>0$, let

$$
\|V\|_{h}=\sum_{\substack{m, n \geq 0 \\ m+n \geq 1}}\left|v_{n, m}\right| h^{n+m}
$$

where

$$
\left|v_{n, m}\right|=\max _{i \in \mathbb{N}_{n+m}} \sup _{X_{i}} \int \prod_{j \neq i} \mathrm{~d} X_{j}\left|v_{n, m}\left(X_{1}, \ldots, X_{n+m}\right)\right| .
$$

Theorem 4.5. Let $C$ be an $(\mathbb{X} \times \mathbb{X})$-matrix, considered as a covariance for a fermionic Gaussian integral, with finite determinant bound $\delta_{C}$ and decay bound $\alpha_{C}$. Denote $\omega_{C}=2 \alpha_{C} \delta_{C}^{-2}$. Let $h>0, h^{\prime}=h+2 \delta_{C}$, and let $V$ be an interaction with $\|V\|_{h^{\prime}}<\infty$. Then the effective action $W(V, C)$, defined as

$$
W(V, C)=\log \int \mathrm{d} \mu_{C}\left(\Psi^{\prime}\right) \mathrm{e}^{V\left(\Psi^{\prime}+\Psi\right)},
$$

exists and is analytic in $V$ : let $W(V, C)=\sum_{p \geq 1} \frac{1}{p !} W_{p}(V, C)$ be the expansion of $W$ in powers of $V$. Then for all $P \geq 1$,

$$
\left\|W(V)-\sum_{p=1}^{P} \frac{1}{p !} W_{p}(V, C)\right\|_{h} \leq \omega_{C} P \frac{\|V\|_{h^{\prime}} P+1}{1-\omega_{C}\|V\|_{h^{\prime}}} .
$$


Proof. Same as in [SW], except that in the bound for the determinants, Lemma 6 of [SW], the Gram constant is replaced by the determinant bound $\delta_{C}$.

The coefficients in the expansion of $W(V, C)(\Psi)$ in the fields $\Psi$ are the amputated connected Green functions, so the above theorem implies their analyticity in the interaction. In particular, analyticity holds for all cases listed in Corollary 4.4, with the appropriate constants. In case of an insulator, the convergence radius is uniform in the temperature. In case of scaled propagators, one obtains power counting bounds that are on all scales operationally equivalent to those with a frequency cutoff. That no $\omega$-cutoff is needed implies that the analytic structure as a function of $\omega$ need not be mutilated in a multiscale construction.

\section{Bounds for the Integration over Large Frequencies}

In a multiscale analysis of many-fermion systems, the integration over fields with large Matsubara frequency is often the first integration step in the analysis. In the following we give bounds for the effective action obtained by this integration step. We first decompose the covariance $C^{(\mathbf{h})}$ given in (14) in an ultraviolet and an infrared part.

Let $\chi_{<}$and $\chi_{>} \in C^{\infty}(\mathbb{R},[0,1])$ with $\chi_{<}+\chi_{>}=1, \chi_{<}(0)=1$, with constants $\kappa>0$ and $\alpha>0$ such that $\chi_{<}(x) \leq \kappa|x|^{-\alpha}$ for all $|x| \geq 1$. Abbreviate the covariance $C_{(\tau, \mathrm{x}),\left(\tau^{\prime}, \mathrm{x}^{\prime}\right)}^{(\mathbf{h})}=C^{(\mathbf{h})}\left(\tau^{\prime}-\tau, \mathrm{x}^{\prime}-\mathrm{x}\right)$. The covariance

$$
C_{\Omega}^{(\mathbf{h},<)}(\tau, \mathrm{x})=\frac{1}{\beta} \sum_{\omega} \int \mu^{a}(\mathrm{dp}) \mathrm{e}^{-\mathrm{i} \omega \tau+\mathrm{ip} \cdot \mathrm{x}} \chi_{<}\left(\frac{\omega}{\Omega}\right) \frac{\mathbf{h}(\mathrm{p})}{\mathrm{i} \omega-E(\mathrm{p})}
$$

is the infrared part of $C^{(\mathbf{h})}$, and

$$
C_{\Omega}^{(\mathbf{h},>)}(\tau, \mathrm{x})=C^{(\mathbf{h})}(\tau, \mathrm{x})-C_{\Omega}^{(\mathbf{h},<)}(\tau, \mathrm{x})
$$

is the ultraviolet part of $C^{(\mathbf{h})}$. An obvious variant of this decomposition is one where the argument of the function $\chi<$ is $\Omega^{-2}\left(\omega^{2}+E(\mathrm{p})^{2}\right)$. Our bounds adapt to this choice in an obvious way, so we will not discuss it further here.

By standard properties of Grassmann Gaussian integration, the convolution with the Gaussian measure $C^{(\mathbf{h})}=C_{\Omega}^{(\mathbf{h},>)}+C_{\Omega}^{(\mathbf{h},<)}$ becomes an iterated convolution, first with $C_{\Omega}^{(\mathbf{h},>)}$, then with $C_{\Omega}^{(\mathbf{h},<)}$ (see, e.g. [S98b]).

\subsection{Determinant bound.}

Lemma 5.1. Let $\chi_{<}$be chosen as above, $\Omega \geq 1$, and $\beta>\pi$. Let $E$ be continuous. Then the determinant bound of $C_{\Omega}^{(\mathbf{h},>)}$ satisfies

$$
\delta_{C_{\Omega}^{(\mathbf{h},>)}}^{2} \leq\|\mathbf{h}\|_{1}\left(K^{\prime}+2 \ln \Omega\right)+\int_{|E(\mathrm{p})| \leq 1} \mu^{a}(\mathrm{dp})|\mathbf{h}(\mathrm{p})| \ln \frac{1}{\|E(\mathrm{p})\| \frac{\pi}{\beta}}
$$

where $K^{\prime}=10+2 \kappa\left(\alpha^{-1}+(\beta \Omega)^{-1}\right)$. 
Proof. By our hypothesis on the decay of $\chi_{<}$, the Fourier transform of the covariance $C_{\Omega}^{(\mathbf{h},<)}$ is $\ell^{1}$ in the Matsubara frequency. Thus $C_{\Omega}^{(\mathbf{h},<)}$ has a Gram representation of type (19), with finite Gram constant $\gamma_{<}$. By (89) and Theorem 1.3, a determinant bound for $C_{\Omega}^{(\mathbf{h},>)}$ is given by $\delta_{C^{(\mathbf{h})}}+\gamma_{<} \cdot \delta_{C^{(\mathbf{h})}}$ was bounded in Corollary 4.2, so it suffices to estimate $\gamma_{<}$. By definition,

$$
\gamma_{<}^{2}=\frac{1}{\beta} \sum_{\omega \in \mathbb{M}_{F}} \int \mu^{a}(\mathrm{dp}) \chi_{<}\left(\frac{\omega}{\Omega}\right) \frac{|\mathbf{h}(\mathrm{p})|}{|\mathrm{i} \omega-E(\mathrm{p})|}
$$

The contribution from $|\omega| \geq 1$ is bounded by

$$
\|\mathbf{h}\|_{1} \frac{1}{\beta} \sum_{\omega \in \mathbb{M}_{F}} \chi_{<}\left(\frac{\omega}{\Omega}\right) \frac{1}{|\omega|} \leq 2\|\mathbf{h}\|_{1}\left(\frac{1}{\pi}+\ln \Omega+\frac{\kappa}{\alpha}+\frac{\kappa}{\beta \Omega}\right) .
$$

For the contribution from $|\omega|<1$, we will repeatedly use the elementary bound $\frac{1}{\beta} \sum_{\omega \in \mathbb{M}_{F}} 1_{|\omega|<u} \leq \frac{2 u}{\pi}$. For $|E(\mathrm{p})| \geq 1,|\mathrm{i} \omega-E(\mathrm{p})|^{-1} \leq 1$, so the contribution from $|\omega|<1$ and $|E(\mathrm{p})| \geq 1$ is bounded by $2\|\mathbf{h}\|_{1} / \pi$. For $|E(\mathrm{p})| \leq 1$, we use that

$$
\frac{1}{\beta} \sum_{\omega \in \mathbb{M}_{F}} 1_{|\omega|<|E(\mathrm{p})|} \frac{1}{|\mathrm{i} \omega-E(\mathrm{p})|} \leq \frac{1}{\beta|E(\mathrm{p})|} \sum_{\omega \in \mathbb{M}_{F}} 1_{|\omega|<|E(\mathrm{p})|} \leq \frac{2}{\pi}
$$

and, bounding the sum by an integral,

$$
\frac{1}{\beta} \sum_{\substack{\omega \in \mathbb{M}_{F} \\|E(\mathrm{p})| \leq|\omega| \leq 1}} \frac{1}{|\mathrm{i} \omega-E(\mathrm{p})|} \leq \frac{1}{\beta} \sum_{\substack{\omega \in \mathbb{M}_{F} \\|E(\mathrm{p})| \leq|\omega| \leq 1}} \frac{1}{|\omega|} \leq \frac{2}{\pi}+\ln \frac{1}{\|E(\mathrm{p})\|_{\frac{\pi}{\beta}}} .
$$

5.2. Decay constant. In this section we show that for a strict cutoff function $\chi_{<}$, and under natural assumptions on the function $E$, the decay constant of $C_{\Omega}^{(\mathbf{h},>)}$ is bounded by a multiple of $\Omega^{-1}$. Thus the extra factor $\log \Omega$ from the determinant bound can be avoided in this bound.

Lemma 5.2. Assume that $\chi_{<}$satisfies $\chi_{<}(x)=1$ for $|x| \leq 1$ and $\chi_{<}(x)=0$ for $|x| \geq 2$. Let $\Omega \geq 1$. Assume that the dispersion function $E$ is the Fourier transform $E=\overline{\hat{F}}$ of some $F \in L^{1}(\Gamma, \mathbb{C})$, and that the inverse Fourier transform $\mathbf{g}$ of $\mathbf{h}$ satifies $\mathbf{g} \in L^{1}(\Gamma, \mathbb{C})$. There is a constant $K>0$, depending only on $\chi_{<}$, such that if $\frac{2 K}{\Omega}\|F\|_{1}<1$ and $\Omega^{-1}\|E\|_{\infty}<1$, the decay constant of $C_{\Omega}^{(\mathbf{h},>)}$ satisfies

$$
\alpha_{C_{\Omega}^{(\mathbf{h},>)}} \leq \frac{K}{\Omega} \frac{\|\mathbf{g}\|_{1}}{1-2 K \Omega^{-1}\|F\|_{1}} .
$$

In particular, if $K\|F\|_{1}<\frac{1}{4} \Omega$, then $\alpha_{C_{\Omega}^{(\mathbf{h},>)}} \leq \frac{2 K}{\Omega}\|\mathbf{g}\|_{1}$. 
Proof. Let

$$
u(\tau)=\frac{1}{\beta} \sum_{\omega \in \frac{\pi}{\beta} \mathbb{Z}} \mathrm{e}^{-\mathrm{i} \omega \tau} \quad \chi<\left(\frac{\omega}{\Omega}\right)
$$

then

$$
C_{\Omega}^{(\mathbf{h},>)}=C^{(\mathbf{h})}-u * C^{(\mathbf{h})},
$$

where the convolution is in $\tau$. By summation by parts,

$$
\left(\mathrm{e}^{\mathrm{i} \frac{\pi}{\beta} \tau}-1\right)^{n} u(\tau)=\frac{1}{\beta} \sum_{\omega} \mathrm{e}^{-\mathrm{i} \omega \tau}\left(\delta^{n} \chi_{<}\right)\left(\frac{\omega}{\Omega}\right),
$$

where $\delta$ is the difference operator $(\delta f)(\omega)=f\left(\omega+\frac{\pi}{\beta}\right)-f(\omega)$. Using that for all $\tau$ with $|\tau| \leq \beta,\left|\mathrm{e}^{\mathrm{i} \frac{\pi}{\beta} \tau}-1\right|=2 \sin \frac{\pi|\tau|}{2 \beta} \geq 2 \frac{|\tau|}{\beta}$ and that $\chi_{<}$is smooth, it follows that

$$
|u(\tau)| \leq \frac{1}{4} K \frac{\Omega}{(1+\Omega|\tau|)^{3}},
$$

where $K$ depends on the sup norms of the first three derivatives of $\chi<$.

Let $a(\tau)=\mathbb{C}(\tau, 0)$. By definition, $a(s)=\theta^{+}(-s)-\frac{1}{2}$, where $\theta^{+}(t)=1$ for $t \geq 1$ and zero otherwise. Because $\int_{-\beta}^{\beta} u(s) \mathrm{d} s=\chi_{<}(0)=1$,

$$
a(\tau)-(u * a)(\tau)=\int_{-\beta}^{\beta} \mathrm{d} s u(s)[a(\tau)-a(\tau-s)] .
$$

The $\frac{1}{2}$ drops out, and $a(\tau)-(u * a)(\tau)=\operatorname{sgn}(\tau) \int_{\mathcal{I}(\tau)} u(s) \mathrm{d} s$, where

$$
\mathcal{I}(\tau)= \begin{cases}{[-\beta,-\beta+\tau] \cup[\tau, \beta]} & \text { for } \tau>0 \\ {[-\beta, \tau] \cup[\beta+\tau, \beta]} & \text { for } \tau \leq 0 .\end{cases}
$$

Our hypothesis on $\mathbf{g}$ and (98) imply that

$$
A_{\Omega}^{(\mathbf{h},>)}(\tau, \mathrm{x})=\mathbf{g}(\mathrm{x})[a(\tau)-(u * a)(\tau)]
$$

satisfies

$$
\left\|A_{\Omega}^{(\mathbf{h},>)}(\tau, \mathrm{x})\right\|_{1} \leq K\|\mathbf{g}\|_{1} \Omega^{-1} .
$$

The same bound holds with $(\mathbf{g}, \mathbf{h})$ replaced by $(F, E)$. For all $(\tau, \mathrm{x})$,

$$
C_{\Omega}^{(\mathbf{h},>)}(\tau, \mathrm{x})=A_{\Omega}^{(\mathbf{h},>)}(\tau, \mathrm{x})+\frac{1}{\beta} \sum_{\omega} \frac{\mathrm{e}^{-\mathrm{i} \omega \tau}}{(\mathrm{i} \omega)^{2}} \chi>\left(\frac{\omega}{\Omega}\right) \int_{\mathcal{B}} \mu^{a}(\mathrm{dp}) \frac{E(\mathrm{p}) \mathbf{h}(\mathrm{p})}{1-\frac{E(\mathrm{p})}{\mathrm{i} \omega}} \mathrm{e}^{\mathrm{ipx}} .
$$

Because $\chi_{>}\left(\frac{\omega}{\Omega}\right)=0$ for $|\omega| \leq \Omega$, the condition $\Omega^{-1}\|E\|_{\infty}<1$ implies that the geometric series for $(1-E(\mathrm{p}) / \mathrm{i} \omega)^{-1}$ converges uniformly in $\mathrm{p}$. By dominated convergence, 
the summation can be exchanged with the integral over $\mathrm{p}$ and the summation over $\omega$. Moreover, by the support properties of $\chi_{<}$, we may insert a factor $\chi_{>}\left(\frac{2 \omega}{\Omega}\right)^{n}$ in the $n^{\text {th }}$ order term in this expansion, to get

$$
C_{\Omega}^{(\mathbf{h},>)}(\tau, \mathrm{x})=A_{\Omega}^{(\mathbf{h},>)}(\tau, \mathrm{x})+\left[\sum_{n=1}^{\infty} A_{\Omega}^{(\mathbf{h},>)} * A_{\Omega / 2}^{(E,>)} * \ldots * A_{\Omega / 2}^{(E,>)}\right](\tau, \mathrm{x}),
$$

where the convolution is in $\tau$ and $\mathrm{x}$ and $n$ factors $A_{\Omega / 2}^{(E,>)}$ appear in the product. The standard $L^{1}$ bound for the convolution implies

$$
\left\|C_{\Omega}^{(\mathbf{h},>)}\right\|_{1} \leq\left\|A_{\Omega}^{(\mathbf{h},>)}\right\|_{1}\left(1+\sum_{n=1}^{\infty}\left\|A_{\Omega / 2}^{(E,>)}\right\|_{1}^{n}\right)
$$

which converges by the hypotheses on $\mathbf{g}, F$, and by (102), and yields the bound (94).

Theorem 4.5 directly applies and implies convergence of the effective action obtained from the integration over large frequencies. Note that because of the way the constants depend on $\Omega$, the initial interaction can be taken arbitrarily strong (as long as it is summable): if $U$ denotes the coupling constant of a quartic interaction, convergence of the expansion for the effective action holds for all $U$ with $\frac{U}{\Omega}(\ln \Omega)^{2}$ small enough, which can always be achieved by taking $\Omega$ large enough. Thus, for arbitrarily strong coupling, the initial integration step is given by a convergent expansion. The consequences and some possible extensions of this are discussed in [S07].

Acknowledgement. This work was supported by DFG grant Sa 1362/2, by the Max-Planck society, and by DFG-Forschergruppe FOR718.

\section{References}

[AGD] Abrikosov, A.A., Gorkov, L.P., Dzyaloshinski, I.E.: Methods of Quantum Field Theory in Statistical Physics. New York: Dover, 1963

[AR] Abdesselam, A., Rivasseau, V.: Explicit fermionic tree expansions. Lett. Math. Phys. 44, 77-88 (1998)

[BR] Bratteli, O., Robinson, D.W.: Operator algebras and quantum statistical mechanics, Vol. 1,2, Berlin-Heidelberg-New York: Springer, 2002

[FKT98] Feldman, J., Knörrer, H., Trubowitz, E.: A representation for fermionic correlation functions. Commun. Math. Phys. 195, 465-493 (1998)

[FKT02] Feldman, J., Knörrer, H., Trubowitz, E.: Fermionic Functional Integrals and the Renormalization Group. CRM Monograph Series, Vol. 16, Providence, RI: Amer. Math. Soc., 2002

[FKT04] Feldman, J., Knörrer, H., Trubowitz, E.: Convergence of pertubation expansions in fermionic models: part 1. Commun. Math. Phys. 247, 195-242 (2004)

[S98a] Salmhofer, M.: Continuous renormalization for fermions and fermi liquid theory. Commun. Math. Phys. 194, 249-295 (1998)

[S98b] Salmhofer, M.: Renormalization. Heidelberg: Springer, 1998

[S07] Salmhofer, M.: Dynamical adjustment of propagators in renormalization group flows. Ann. Phys. (Leipzig) 16(3), 171-206 (2007)

[SW] Salmhofer, M., Wieczerkowski, C.: Positivity and convergence in fermionic quantum field theory. J. Stat. Phys. 99, 557-586 (2000)

[PS] Pedra, W., Salmhofer, M.: On the Mathematical Theory of Fermi Liquids in Two Dimensions. To appear

[St] Stein, E.: Harmonic Analysis. Chapter 3; Princeton, NJ: Princeton University Press, 1993 